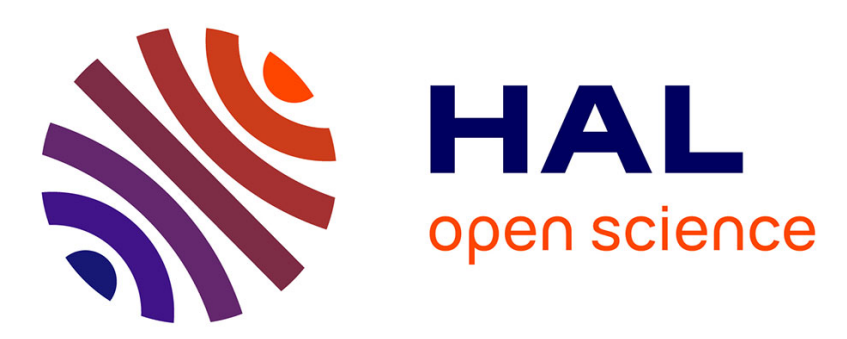

\title{
Miniaturized Soft Transformable Swimmer for Evironmentally Friendly and Sustainable Fluidic Carrier
} Gilgueng Hwang, Atsushi Toyokura, Akio Higo, Beomjoon Kim, Yoshio Mita

\section{To cite this version:}

Gilgueng Hwang, Atsushi Toyokura, Akio Higo, Beomjoon Kim, Yoshio Mita. Miniaturized Soft Transformable Swimmer for Evironmentally Friendly and Sustainable Fluidic Carrier. 2021 Symposium on Design, Test, Integration \& Packaging of MEMS and MOEMS (DTIP), Aug 2021, Paris, France. pp.1-4, 10.1109/DTIP54218.2021.9568678 . hal-03426152

\section{HAL Id: hal-03426152 https://hal.science/hal-03426152}

Submitted on 12 Nov 2021

HAL is a multi-disciplinary open access archive for the deposit and dissemination of scientific research documents, whether they are published or not. The documents may come from teaching and research institutions in France or abroad, or from public or private research centers.
L'archive ouverte pluridisciplinaire HAL, est destinée au dépôt et à la diffusion de documents scientifiques de niveau recherche, publiés ou non, émanant des établissements d'enseignement et de recherche français ou étrangers, des laboratoires publics ou privés. 


\section{Miniaturized Soft Transformable Swimmer for Evironmentally Friendly and Sustainable Fluidic Carrier}

\author{
Gilgueng Hwang* \\ LIMMS-CNRS, Institute of Industrial \\ Science, The Unversity of Tokyo \\ Dept. of Electrical Engineering and \\ Information Science, The University of \\ Tokyo, Tokyo, JAPAN \\ C2N-CNRS, Université Paris-Saclay, \\ Palaiseau, FRANCE \\ hwang@if.t.u-tokyo.ac.jp \\ Beomjoon Kim \\ LIMMS-CNRS, Institute of Industrial \\ Science, The University of Tokyo, \\ Tokyo, JAPAN \\ bjoonkim@iis.u-tokyo.ac.jp
}

\author{
Atsushi Toyokura \\ Dept. of Electrical Engineering and \\ Information Science, The University of \\ Tokyo, Tokyo, JAPAN \\ Systems Design Lab, The University of \\ Tokyo, Tokyo, JAPAN \\ toyokura@if.t.u-tokyo.ac.jp \\ Yoshio Mita \\ LIMMS-CNRS, Institute of Industrial \\ Science, The Unversity of Tokyo \\ Dept. of Electrical Engineering and \\ Information Science, The University of \\ Tokyo, Tokyo, JAPAN, \\ Systems Design Lab, The University of \\ Tokyo, Tokyo, JAPAN \\ mita@ee.t.u-tokyo.ac.jp
}

\author{
Akio Higo \\ Systems Design Lab, \\ The University of Tokyo, Tokyo, \\ JAPAN \\ higo@if.t.u-tokyo.ac.jp
}

\begin{abstract}
We developed a magnetically actuated soft transformable microswimmer for an environmentally friendly and sustainable fluidic handling. They are made of the composite mixture of magnetic micro particles and soft UV curable resin. The custom developed manufacturing system allows to program the local magnetizations of each fractional areas with UV light exposure for both the bending motion by magnetic field and the surface microstructuring to minimize surface stiction. The manufacturable device scale ranges from the smallest of $385 \times 702 \mu \mathrm{m}^{2}$ to the largest $2.0 \times 5.5 \mathrm{~mm}^{2}$. We demonstrated successful liquid sample handling between droplets or hydrogel substrates showing a great potential to serve as a fluidic carrier for biochemical sample analysis or drug delivery.
\end{abstract}

Keywords-Soft transformable microswimmer, Surface structuring, Fluidic manipulation

\section{INTRODUCTION}

Biological or chemical sample handling is one of the most essential tasks in the laboratories for sample preparations and diagnostics. These are currently being done by manual or robotic pipetting. Even if the operators are handy, such pipetting tasks always have the risk of contaminations for both operator and sample. In addition, handling the sub-microliter samples requires precise fluidic control systems such as syringe pump or pressure controller thus it is mostly limited to the laboratory environments. For the field bioanalysis or remote drug delivery, it is crucial to expand the workspace to the field environments out of laboratory. Miniaturized swimmer could be an alternative to such tasks. Microswimmers opened new biomedical applications like microsurgery, drug delivery, hyperthermia, oxygen sensing or other precision tasks [1]. They can also be used as wireless in-

This work was supported in part by JSPS KAKENHI Grant Number 21K03931 and ANR Grant Number ANR-16-CE33-0022. vitro microfluidic manipulators for mechanical force sensing [2,3], rheological sensing [4] or as in-vitro fertilization systems $[5,6]$. Recent progress on the mobile micro devices showed their great potentials either to the solid sample transport [7] or to the liquid sample handling [8]. However their manufacturing complexities and fragilities do not guarantee the sustainable motions in between multi-phase (liquid/air) environments. Although those rigid body microswimmers have opened many promising biomedical applications, their practical applications still require much more extensive studies particularly to enhance the environmental adaptabilities and the integrated functionalities.

Getting closer to their real-life applications, soft body mobile micro devices have drawn much interest to biomedical or microfluidic applications [9,10]. Morphologically programmable soft microswimmers could adapt themselves better to navigate through complex environments thus they could be promising candidates for the environmentally friendly and sustainable fluidic carriers [11,12]. Various actuation principles have been studied. Among the physical actuation principles, thermal actuation is one of the most commonly studied method for the self-deforming structures. The local heating to the structures could be generated either by absorbing light $[13,14]$ or by applying electrical currents [15]. This has also been extensively studied at microscale [16]. However, the actuation frequency by thermal energy is slow due to the requirement of long time for heating and cooling. Moreover, reversable actuation requires much complex designs of multiple structure layers. Compared to the heat radiation efficiency in air or vacuum, the thermal actuation drastically loses its efficiency in water for biological or chemical applications.

Alternatively magnetic actuation could be considered for soft actuation in wet environments thanks to the fact that 


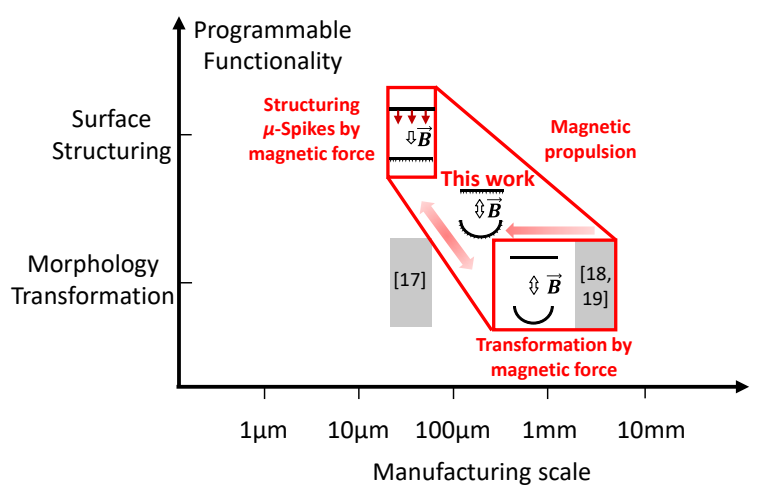

Figure 1: Soft transformable magnetic microswimmers with morphology and surface programmability. Grey areas indicate other works.

external magnetic field does not occur chemical reaction in the liquid environment being transparent to wet solutions.

However, to achieve the morphological transformation by magnetic actuation, several sophisticated manufacturing processes are required especially for the programming of the local magnetizations of the structure. So far the manufacturable scale has been mainly focused on the narrow scale ranges either in micrometers or in millimeters [17-19] (Fig. 1). In the micro scale device, the nanoscale patterning of magnetic materials has been demonstrated for the morphological transforming structure of a few tens of micrometer scale [17]. Millimetric scale morphological transformation was fabricated by discrete magnetizations $[18,19]$. These opened new manufacturing possibilities of programming the local magnetizations for the morphological transformation by external magnetic field. The remaining challenges are to further enhance the programmable functionalities and also to enlarge the scale range of device. In this work, we aim to develop a soft microswimmer with manufacturable scale range from micro to millimeter for environmentally friendly and sustainable fluidic handling.

In addition to the morphological transformability, the programmability of surface structuring is also important to enhance the multi-phase environment adaptability for biological sample handling and medical diagnostics. Soft microswimmers with only morphological transformability could suffer from high surface tension at water-to-air interface thus their potential applications could be limited to completely immersed aquatic condition. Major limitation of such thin-film soft microswimmers is their vulnerable propulsion characteristics at the liquid/air or liquid/solid interfaces due to their lacking capabilities to overcome both the high surface tension of liquid/air and the high stiction between two solids. Therefore, it is crucial to enhance the propulsion robustness and the wet/dry environment compatibility.

In this context, we consider that there is a potential interest to further increase the level of programmable functionality and to further enlarge the range of manufacturing scale. Figure 1 illustrates the relation between the manufacturing scale range in the $\mathrm{X}$-axis and the level of programmable functionality (morphology transformation and surface structuring) of soft microswimmers in the Y-axis.

In this paper, we aim to develop a multiscale magnetooptical manufacturing platform for the fabrication of multiphysics soft microswimmers with programmable surface and morphology. We will demonstrate the programming of the local magnetizations for the morphological transformation such as grasping/bending actuation and also the structuring of the magnetic micro spikes on the surface of microswimmers to increase the multienvironment compatibility.

\section{MethodS AND System}

\section{A. Device concept and manufacturing process}

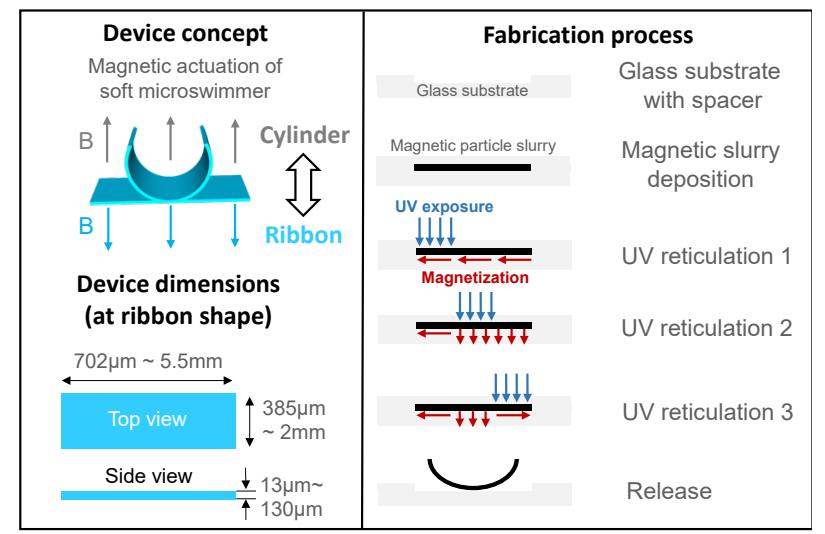

Figure 2: Device concept, dimensions and fabrication process of soft microswimmer with morphology transformation between ribbon and cylinder shape.

Fig. 2 shows the proposed device concept of the soft microswimmer which can self-deform by external magnetic fields. This can be achieved by the fabrication process of programming local magnetizations. The magnetic slurry composed of magnetic particles and ultraviolet (UV) sensitive soft resin is deposited onto the depth controlled chamber (depth from $13 \mu \mathrm{m}$ to $130 \mu \mathrm{m}$ ). Then sequential UV light exposures are made with controlling magnetic field direction using the custom developed manufacturing system shown in the Fig. 3. After rinsing the sample by isopropyl alcohol, a drying process allows to release the reticulated soft microswimmers.

Once the optical system calibrations are completed, we attempt to fabricate soft microswimmers with simple ribbon shapes. The device concept and the fabrication process for such soft microswimmers are shown in the Figure 2. The ribbon shape soft microswimmers are intended to bend along its axis by the applied external magnetic field. The fabrication is made by four steps which consist of the local exposures of each three sub-sections with different magnetic flux directions and the last global exposure of the whole ribbon shape to confirm the reticulation without applying external magnetic field. Prior to the exposure steps of each sub-sections, the direction of magnetic flux is modified to align the magnetic particles thus to program the local magnetizations. Figure 2 shows the fabrication process and the photos of the UV exposures of each sub-section. Posterior to the UV exposure step, the ribbon shape with UV exposures is developed and rinsed with isopropyl alcohol solution. The fabricated structure is measured to be $2.03 \mathrm{~mm}$ by $5.52 \mathrm{~mm}$ from the optical microscopy. Being close to the dimension of the exposure pattern generated from computer $(2 \mathrm{~mm}$ by $5.5 \mathrm{~mm})$, the alignment of the optical system and the calibration between the input pattern models and the patterns with UV exposures are confirmed. 


\section{B. Manufacturing system overview}

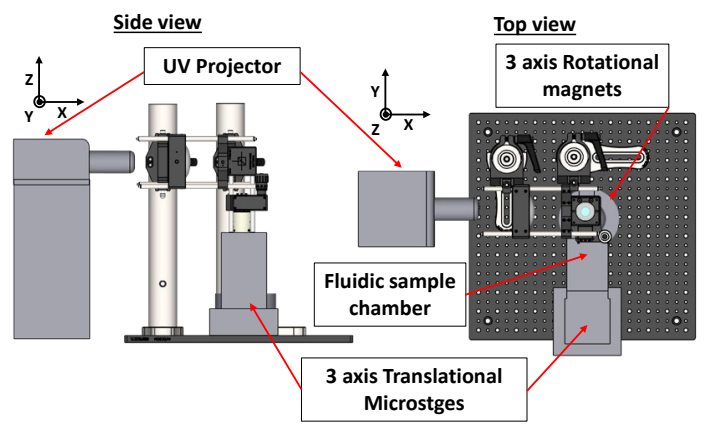

Figure 3: Manufacturing system for soft microswimmer.

The magneto-optical manufacturing system for the rapid prototyping of soft microswimmers is shown in the Figure 3. The system consists of the two main parts which are the UV projector and the magnetization direction control system. The UV projector (LC4500-UV M8, Keynote Technologies, LLC) is used for the source of UV light illumination through the optical system. 1-inch cube Neodymium permanent magnet is inserted between the two hemispherical shape housings. The assembled sphere with magnet is mounted on the hollow cylindrical support fixed to the base optical table. The direction of magnetic flux can be modified manually by rotating the sphere with magnet in any rotations. Magnetic slurry sample is prepared with the mixture (mass ratio 1:1) of the magnetic microparticles and the UV (405 nm wavelength) curable soft resin. The prepared magnetic slurry is filled into the chamber with the depth of $130 \mu \mathrm{m}$ which can be adjusted by the thickness of spacer. The chamber is covered with a cover glass to be able to maintain the thickness of the magnetic slurry same as the height of the chamber. The sample with the magnetic slurry is mounted onto 3 axis micro stage then placed in between the lens objective of the optical system and the sphere with magnetization direction control. The system is covered with UV protection case which can protect UV sensitive samples from both the external light and the dusts. Installing a CCD camera allows to monitor the manufacturing process via external computer screen to avoid unnecessary UV exposure to the operator's eye. The manufacturing can also be made outside the cleanroom.

\section{RESULTS}

In this section, first we show the major characteristics of the manufacturing system which includes the multiscale manufacturing and the surface micro structuring. Then we show the morphology bending by external magnetic field. Finally, we demonstrate the liquid sample handling functionalities of the soft microswimmers between the droplets or to hydrogel substrate.

\section{A. Multiscale manufacturing}

To analyze the manufacturing scalability of the soft microswimmer manufacturing system without changing the current configuration of the optical system, we performed to manufacture 6 different scale models with the same ribbon shape. The scales of the fabricated soft microswimmers range from the smallest one $(385 \mu \mathrm{m} \times 702 \mu \mathrm{m} \times 13 \mu \mathrm{m})$ to the largest one $(2030 \mu \mathrm{m} \times 5520 \mu \mathrm{m} \times 130 \mu \mathrm{m})$. Smaller or larger structures could also be manufactured by modifying the optical system. For example, incorporating the higher resolution UV projector and the higher magnification objective lens should allow the fabrication of further smaller structures. In addition to scaling the lateral dimensions, the thickness of the structure can also be reduced by using the sample chambers with shallow depths. It should be noted here that the manufacturable workspace area at single process step is around $1 \mathrm{~cm}^{2}$. However, enlarging the manufacturable surface area could also be made to multiply the numbers of structures array or to enlarge the dimensions of the structure. This can be achieved by using larger workspace motion stage and the larger sample chamber.

\section{B. Surface micro structuring}
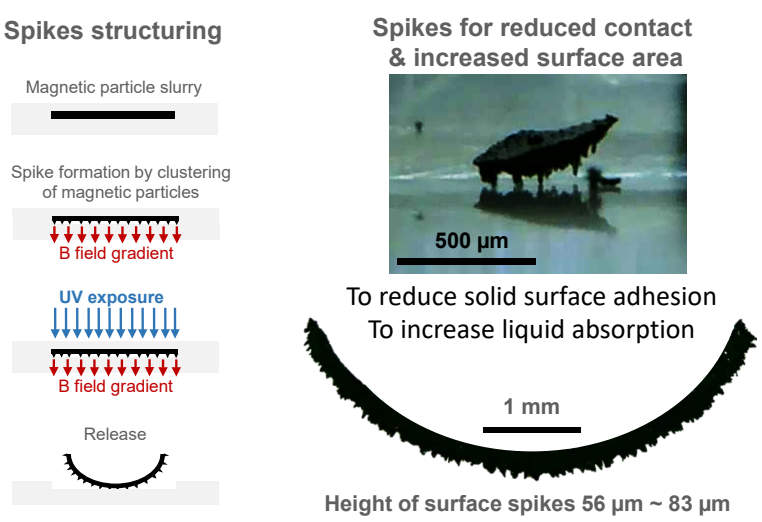

Figure 4: Surface structuring of micro spikes.

The manufacturing system also allows to structure the surface with micro spikes. As shown in the Figure 4, magnetic slurry composed of ferromagnetic microparticles is attracted downward to external magnetic field in vertical direction to form the micro spikes. The reticulation by UV light exposure will allow to solidify the micro spikes and the support ribbon to form the soft microswimmers with micro spikes. Such physically functionalized surface contributes to decrease the contact area to the substrate which can help to overcome excessive surface stiction. The micro spikes are crucial to reduce the surface stiction and the surface tension at solid-liquid-air interfaces thus to facilitate the propulsion between droplets. In addition, such micro spikes increase the

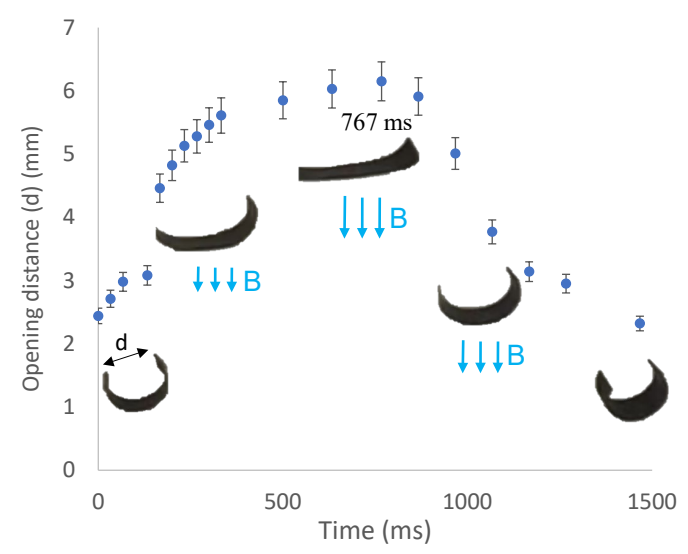

Figure 5: Soft microswimmer with bending and unbending by external magnetic field. Scale of the soft microswimmer is $2 \mathrm{~mm} \times 6 \mathrm{~mm} \times 130 \mu \mathrm{m}$. 
surface-to-volume ratio of soft microswimmers thus to increase the absorbed liquid volume to carry. The absorbed liquid by soft microswimmer serves to lubricate the motion on a dry substrate between multiple droplets.

\section{Bending actuation and liquid sample handling}

Morphology deformation by bending is the most basic function of the soft microswimmer. This is an important feature for stable handlings of solid objects useful for drug delivery or manipulation of biological cells/tissues. Figure 5 shows the morphological transformation by magnetic actuation of soft microswimmer. In the absence of external magnetic field, the soft microswimmers remain to be bent. With the application of external magnetic field downward vertical direction, the soft microswimmer unbends close to the flat ribbon structure (at $767 \mathrm{~ms}$ ). Cutting external magnetic field bends the soft microswimmer close to the initial status due to the elasticity. Considering the opening distances of the swimmer ranging from $2.5 \mathrm{~mm}$ to $6 \mathrm{~mm}$, few millimeters scale solid object samples with arbitrary shapes could be handled by this magnetically actuated soft microswimmers.

The surface and morphologically functional soft microswimmers are promising to serve as a mobile liquid carrier. We demonstrate liquid handling from droplet to hydrogel substrate. A soft microswimmer with the dimension of $2 \mathrm{~mm}$ by $5.5 \mathrm{~mm}$ moved from dry substrate to a red dye droplet by magnetic actuation setup shown in Figure 6 . The rotational motion is generated by 3 axis Helmholtz coils and the translational motion and the bending motion are generated by the gradient coil mounted to the motion stage similar to other works $[3,4]$. The soft microswimmer absorbs the red dye thanks to the high surface-to-volume ratio of micro spikes. Rotating magnetic field could induce the rotating motion for enhancing the absorption by mixing inside droplet. The soft microswimmer with red dye absorption moves out of the droplet to a hydrogel substrate. Vertical magnetic field is applied by gradient coil to press the soft microswimmer onto the hydrogel substrate to accelerate the release of red dye. Alternatively, the soft microswimmer can also move to another water droplet to mix two liquids by rotational motion.

\section{CONClusions}

The current system allows to fabricate the soft microswimmers from the smallest of $385 \times 702 \mu \mathrm{m}^{2}$ to the largest of $2.0 \times 5.5 \mathrm{~mm}^{2}$. The system also allows surface microstructuring of the spikes with the height amplitudes between 56 and 83 microns. This is an useful feature to be able to minimize the surface stiction thus to guarantee the sustainable manipulation. We also demonstrated surface bending by magnetic field which also allows physical adaptation to further enhance the environment adaptability and sustainability. Finally we have demonstrated swimming inside liquid chamber under external magnetic field controls for uniform rotating magnetic field and magnetic field gradient. They are able to transport liquid sample between droplets or from droplet to hydrogel substrate (video). Furthermore the soft microswimmers can also transport solid samples in a confined fluidic environment to simulate their potential clinical applications such as biopsy or drug delivery.

CONTACT: * G. Hwang, hwang@if.t.u-tokyo.ac.jp

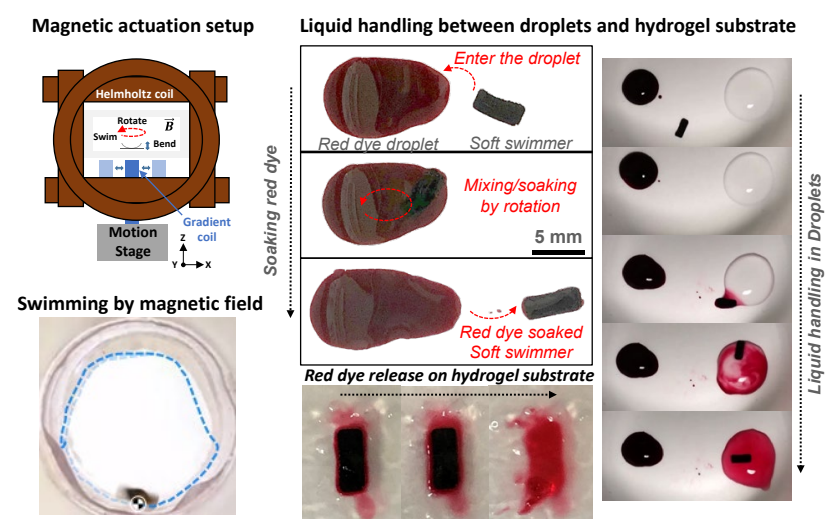

Figure 6: Magnetic actuation of soft microswimmers and liquid sample handlings between droplets or to hydrogel substrate. Scale of microswimmer $2 \mathrm{~mm} \times 5.5 \mathrm{~mm} \times 130 \mu \mathrm{m}$.

[1] B. J. Nelson, I. K. Kaliakatsos, J. J. Abbott, "Microrobots for Minimally Invasive Medicine”, Annu. Rev. Biomed. Eng., vol. 12, no. 1, pp. 55-85, 2010.

[2] T. Kawahara, M. Sugita, M. Hagiwara, F. Arai, H. Kawano, I. ShihiraIshikawa, A. Miyawaki, "On-chip microrobot for investigating the response of aquatic microorganisms to mechanical stimulation", Lab Chip, vol. 13, pp. 1070-1078, 2013.

[3] G. Hwang, I. A. Ivan, J. Agnus, H. Salmon, S. Alvo, N. Chaillet, S. Regnier, A.-M. Haghiri-Gosnet, "Mobile microrobotic manipulator in microfluidics”, Sens. Act. A: Phys., vol. 215, pp. 56-64, 2014.

[4] H. Salmon, L. Couraud, G. Hwang, "On-chip mobile microrobotic transducer for high-temporal resolution sensing using dynamics analysis", Sens. Act. A: Phys., vol. 288, pp. 27-38, 2019.

[5] V. Magdanz, S. Sanchez, O. G. Schmidt, "Development of a SpermFlagella Driven Micro-Bio-Robot", Adv. Mat., vol. 25, no. 45, pp. 6581-6588, 2013.

[6] M. Medina-Sanchez, L. Schwarz, A. K. Meyer, F. Hebenstreit, O. G. Schmidt, "Cellular Cargo Delivery: Toward Assisted Fertilization by Sperm Carrying Micromotors", Nano Lett., vol. 16, no. 1, pp. 555-561.

[7] G. Hwang, A. Mizushima, E. Lebrasseur, K. Misumi, N. Usami, A. Higo, Y. Mita, Sens. Act. A: Phys., 318, 112502, 2021.

[8] D. Decanini, A. Harouri, Y. Mita, B. J. Kim, G. Hwang, AIP Rev. Sci. Instrum. 91, 086104, 2020.

[9] C. Hu, S. Pane, B. J. Nelson, "Soft Micro- and Nanorobotics", Annu. Rev. Cont. Rob. Auto. Syst., vol. 1, pp. 53-75, 2018.

[10] M. Sitti, "Miniature soft robots - road to the clinic", Nat. Rev. Mat., vol. 3, pp. 74-75, 2018.

[11] H.-W. Huang, F. E. Uslu, P. Katsamba, E. Lauga, M. S. Sakar, B. J. Nelson, "Adaptive locomotion of artificial microswimmers", Sci. Adv., vol. 5, no. 1, pp. eaau1532, 2019.

[12] L. Pancaldi, P. Dirix, A. Fanelli, A. M. Lima, N. Stergiopulos, P. J. Mosimann, D. Ghezzi, M. S. Sakar, "Flow driven robotic navigation of microengineered endovascular probes", Nat. Comm., vol. 11, pp. 6356, 2020.

[13] Y. Liu, B. Shaw, M. D. Dickey, J. Genzer, "Sequential self-folding of polymer sheets", Sci. Adv., vol. 3, no. 3, pp. e1602417, 2017.

[14] J. Mu, C. Hou, H. Wang, Y. Li, Q. Zhang, M. Zhu, "Origami-inspired active graphene-based paper for programmable instant self-folding walking devices", Sci. Adv., vol. 1, no. 10, pp. e1500533, 2015.

[15] S. Felton, M. Tolley, E. Demaine, D. Rus, R. Wood, "A method for building self-folding machines", Science, vol. 345 , no. 6197, pp. 644646, 2014.

[16] D. George, M. J. Madou, Origami MEMS, Springer, 2021.

[17] J. Cui, T.-Y. Huang, Z. Luo, P. Testa, H. Gu, X.-Z. Chen, B. J. Nelson, L. J. Heyderman, "Nanomagnetic encoding of shape-morphing micromachines", Nature, vol. 575, no. 7781, pp. 164-168, 2019.

[18] T. Xu, J. Zhang, M. Salehizadeh, O. Onaizah, E. Diller, "Millimeterscale flexible robots with programmable three-dimensional magnetization and motions", Sci. Rob., vol. 4, no. 29, pp. eaav4494, 2019.

[19] Z. Ren, W. Xu, Z. Dong, M. Sitti, "Multi-functional soft-bodied jellyfish-like swimming", Nat. Comm., vol. 10, no. 2703, 2019. 